\title{
Nano/microparticle Formulations for Universal Influenza Vaccines
}

\author{
Dylan A. Hendy, ${ }^{1}$ Eva A. Amouzougan, ${ }^{1}$ Isabella C. Young, ${ }^{1}$ Eric M. Bachelder, ${ }^{1}$ and Kristy M. Ainslie ${ }^{1,2,3,4}{ }_{(\mathbb{C}}$
}

Received 8 October 2021; accepted 17 December 2021; published online 7January 2022

\begin{abstract}
Influenza affects millions of people worldwide and can result in severe sickness and even death. The best method of prevention is vaccination; however, the seasonal influenza vaccine often suffers from low efficacy and requires yearly vaccination due to changes in strain and viral mutations. More conserved universal influenza antigens like M2 ectodomain (M2e) and the stalk region of hemagglutinin (HA stalk) have been used clinically but often suffer from low antigenicity. To increase antigenicity, universal antigens have been formulated using nano/microparticles as vaccine carriers against influenza. Utilizing polymers, liposomes, metal, and protein-based particles, indicators of immunity and protection in mouse, pig, ferrets, and chicken models of influenza have been shown. In this review, seasonal and universal influenza vaccine formulations comprised of these materials including their physiochemical properties, fabrication, characterization, and biologic responses in vivo are highlighted. The review is concluded with future perspectives for nano/microparticles as carrier systems and other considerations within the universal influenza vaccine delivery landscape.
\end{abstract}

KEY WORDS: polymeric nanoparticles; liposomes; gold nanoparticles; protein nanoparticles; flu.

\section{INTRODUCTION}

According to the World Health Organization (WHO), there are up to 5 million severe cases and 500,000 deaths per year due to influenza. In the USA alone, the total economic burden for influenza is $\$ 87.1$ billion per year, which accounts for hospitalization visits, out-patient care, loss of earnings, and loss of life due to this epidemic (1). Individuals over the age of 65 , infants, pregnant women, and those living in lowincome countries are among the most susceptible to severe and lethal influenza infections (2). The Centers for Disease Control (CDC) identifies four types of influenza virus: A, B, $\mathrm{C}$, and D. Influenza $\mathrm{A}$ virus (IAV) and $\mathrm{B}$ are the most common seasonal epidemics that circulate in the USA with IAV causing the utmost threat to human health because it has both human and animal hosts, whereas influenza B only

\footnotetext{
${ }^{1}$ Division of Pharmacoengineering and Molecular Pharmaceutics, Eshelman School of Pharmacy, University of North Carolina at Chapel Hill, 4012 Marsico Hall, 125 Mason Farm Road, Chapel Hill, North Carolina 27599, USA.

${ }^{2}$ Joint Department of Biomedical Engineering, University of North Carolina at Chapel Hill and North Carolina State University, Chapel Hill, North Carolina, USA.

${ }^{3}$ Department of Microbiology and Immunology, UNC School of Medicine, University of North Carolina, Chapel Hill, North Carolina, USA.

${ }^{4}$ To whom correspondence should be addressed. (e-mail: ainsliek@email.unc.edu)
}

infects humans (3). IAV can be divided into subtypes based on the surface proteins hemagglutinin (HA) and neuraminidase (NA) (4). The most common subtypes found in the population are $\mathrm{H} 1 \mathrm{~N} 1$ and $\mathrm{H} 3 \mathrm{~N} 2$. The specific nomenclature of each virus contains antigenic type, geographical location, strain number, and year of isolation, such as A/California/07/ 2009 (H1N1) or A/Victoria/210/2009 (H3N2) (4).

Typical IAV vaccines are defined as seasonal or universal. Seasonal influenza vaccines can achieve a single year of protection from the infection. While seasonal vaccines can provide protection to high-risk groups, the continuous antigenic drift of influenza subtypes complicates effective vaccine production. This antigenic drift is caused by mutations in membrane proteins HA and NA. When these mutations occur, the seasonal vaccine will not match the circulating epidemic virus strain and the vaccine will lose its effectiveness.

In contrast to a seasonal influenza vaccine, a universal influenza vaccine can have broad protection against multiple subtypes or strains of the virus. They are, however, significantly more difficult to develop than seasonal influenza vaccine since they ideally provide protection independent of antigenic drift of HA/NA subtypes (5). To accomplish this, universal vaccines use antigens that are more conserved across subtypes such as HA stalk (also called stem), and the ectodomain of matrix protein 2 (M2e) (Figure 1). However, these universal influenza antigens are often weakly immunogenic and require immune activating adjuvants and/or 
advanced formulation to elicit a protective immunity. As such, universal influenza vaccines have been formulated using nano/microparticles (NPs).

Using NPs as a vaccine carrier system can protect vaccine molecules from premature protease degradation, improve stability, elicit sustainable release, and assist in targeted delivery of an immunogen to antigen-presenting cells (APCs) (6). Due to these attributes, in many cases, nanoparticles can also act as a vaccine adjuvant (7). This review will highlight particulate platforms that are used for seasonal (Table I) and universal (Table II) influenza vaccines. NPs will be divided into those comprised of polymers, liposomes, metals, and proteins (Figure 2). To preface the discussion of these platforms, we will discuss characterization of the NPs, example use as a seasonal and universal influenza vaccine, and vaccine efficacy in vivo.

\section{CHARACTERIZATION OF NANOPARTICLES}

When comparing NPs, there are several physiochemical properties that can vary from platform to platform, but also influence how the NPs interact with APCs and other immune cells (22). Of the physiochemical characterization of NPs for vaccines, surface charge, particle size, loading capacity of the particles, and drug release are the most commonly reported.

Surface charge is commonly conveyed as zeta potential ( $\zeta$-potential). $\zeta$-Potential represents the electric potential of the surface of the particles when suspended in a liquid and can indicate the stability of the particle in suspension as well as interactions with cells. Potentials of approximately positive or negative $40-50 \mathrm{mV}$ represent good colloidal stability, wherein the particles are repelling enough from each other that they stably suspend. Particles with a positive charge can non-specifically interact with the negatively charged cell surface and have increased opsonin binding and therefore greater clearance than anionic particles (23). Furthermore, positively charged particles can better interact with nucleic acid for the delivery of mRNA vaccines. Most $\zeta$-potential measurements are performed in tandem with dynamic light scattering (DLS) measurements.
DLS is one very common method to measure particle size in suspension (hydrodynamic radius). In determining DLS measurements, intensity distributions naturally weigh particle diameter according to the scattering intensity of each particle fraction and account for aggregation of particles. Intensity distributions can be more accurate alone than volume and density distributions which are relative and assume the particles are spherical and homogenously dispersed. Often with DLS, a polydispersity index (PDI) is calculated where values $<0.05$ indicate highly monodisperse particles and generally a PDI greater than 0.7 is too broad for accurate measurement by DLS. In addition to DLS, micrographs such as those generated by scanning electron microscopy (SEM), transmission electron microscopy (TEM), or atomic force microscopy (AFM) can be used to determine size. Although these imaging techniques are often performed under a vacuum and not with a hydrated particle, there are also environmental SEM as well as AFM which can be performed, albeit with a smaller measurement range than typically seen under vacuum. Overall, size is a particularly important physicochemical property in the context of vaccine delivery since particles of certain sizes are more efficiently phagocytosed by APCs (24).

As important as NP size is the loading of the antigen or adjuvant associated with the particle. Antigens and adjuvants are often encapsulated in NPs, covalently bound to the surface of the NPs, or adsorbed to the surface if the NPs via electrostatic interactions. Often the actual loading of the antigens associated with the particles is determined by methods like absorbance, fluorescence, and highperformance liquid chromatography (HPLC), and for protein-based antigen a detection assay (e.g., bicinchoninic acid (BCA), fluorescamine, enzyme-linked immunosorbent assay (ELISA)). For the encapsulation of mRNA, loading can be determined by real-time reverse transcription polymerase chain reaction (RT-qPCR). Since encapsulation or covalent binding chemistry is not $100 \%$ effective, there is an encapsulation efficiency (EE) measurement that is determined from the actual loading divided by the theoretical loading. For generation of particles through emulsion

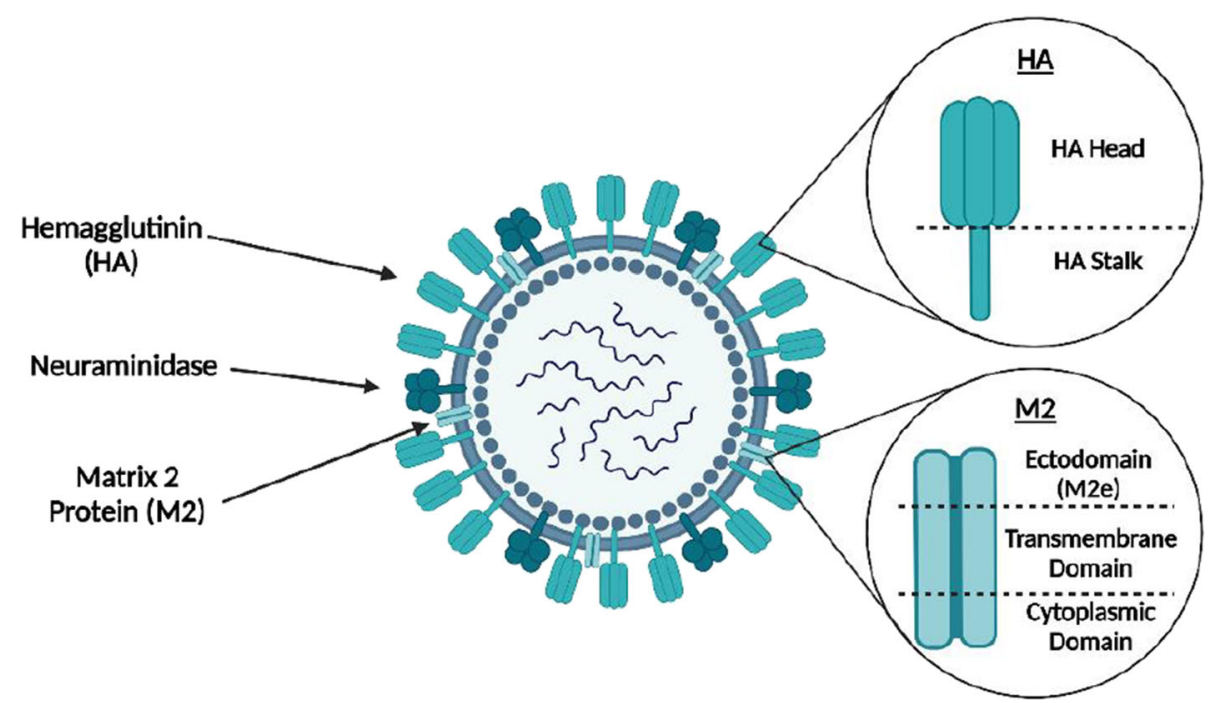

Figure 1. Generalized diagram of influenza A virus (IAV). Labeled are different proteins that have been investigated as potential antigens for universal influenza vaccinations 
processes (e.g., poly lactic-co-glycolic acid (PLGA) NPs), the EE can vary based on the physiochemical properties of the encapsulate and its ability to diffuse into the continuous water phase. For covalently bound NPs, antigen loading is dependent on the chemistry used to functionalize the antigen and it is important to wash away the unreacted antigen before measurement. Regardless of encapsulation or covalently bound antigen capture, valid background values are needed to determine actual loading. This can be as simple as a blank or vehicle control; however, for surface functionalized NPs, it is important to determine the value of antigen non-specifically adsorbed on a blank particle. Besides loading, the release of encapsulate from the NP can help to relate the kinetics of the antigen or adjuvant. Often using the same techniques as performed for loading studies, the amount of antigen or adjuvant released or retained can be measured, when NPs are placed in sink conditions, typically using a dialysis system.

An important in vitro characterization of the released and associated antigen or adjuvant is to determine if it is biologically active and performs the function it should. For adjuvant activity, simple innate immune assays wherein immortalized (e.g., RAW macrophages) or primary cells (e.g., bone marrow derived dendritic cells (BMDCs)) can be cultured with NPs or positive control (e.g., LPS), and the supernatants assayed for cytokine activity via ELISA. An important control in the evaluation of innate activity is a vehicle-only control since high production of cytokines can indicate endotoxin contamination. Endotoxin contamination cannot be removed with filtering, but can be easily determined by absorbance with the limulus amebocyte lysate (LAL) test that can quantitatively measure the amount of endotoxin in a sample (22). Uptake studies by APCs are also an important in vitro assay that is best performed using fluorescent dyes (e.g., fluorescence activated cell sorting/ scanning (FACS)) and confocal microscopy $(25,26)$. Despite efforts washing the NPs off, other methods of uptake such as flow cytometry and epifluorescence only indicate particle association with cells and will not indicate actual uptake unless a dye which changes excitation and emission when internalization is used. To characterize antigen presentation in an in vitro setting, transgenic $\mathrm{T}$ cell lines such as B3Z and KZO, developed by Dr. Nilabh Shastri, can be used to illustrate MHC I and MHC II presentation of model antigen ovalbumin (OVA), respectively $(27,28)$. However, presentation to $\mathrm{T}$ cells relies on peptide presentation and may not indicate the correct tertiary protein structure of the antigen, which is needed for optimal B cell activation and generation of neutralizing antibodies. The use of high energy mixing (e.g., sonication, homogenization), contact with solvent, and other environmental factors during NP fabrication can lead to protein denaturation and reduced neutralizing antibody titer (29). Characterization of expressed antigen by circular dichroism, Fourier-transformed infrared (FTIR) spectroscopy, or antibody binding may be needed to understand antigen structure better.

\section{VACCINE EFFICACY IN VIVO}

Once the particles are formulated and optimized with the antigen and/or adjuvant, in vivo testing to assess the efficacy of the vaccine is essential. Mouse models are most common in 


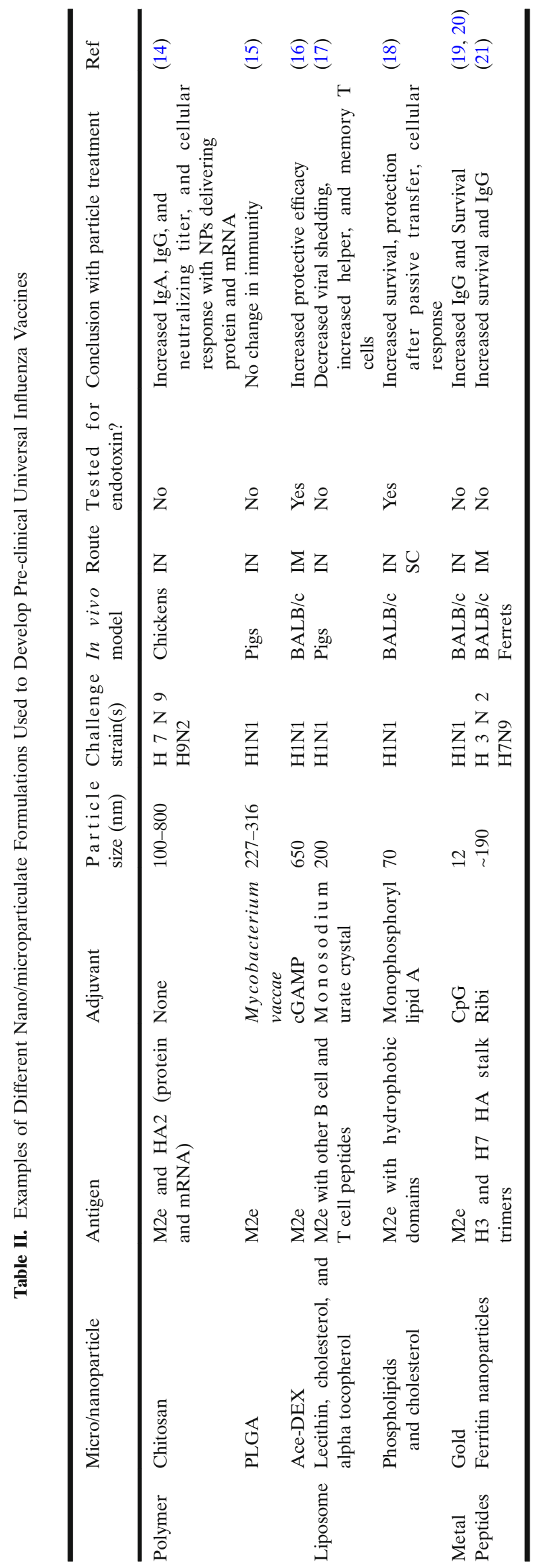

influenza vaccine testing; however, pigs, ferrets, and chickens have also been used to evaluate NP universal influenza vaccines, with ferrets being the most applicable larger animal model for influenza. Of the mice models used, BALB/c are the most reported for evaluation of NPs with M2e because they are reported to be the most effective for that peptide (30).

With regard to vaccine schedule, most vaccine formulations will have a prime, and at least one boost. Another important aspect in vaccine research is the administration route, with needle-free (e.g., intranasal (IN), and oral) preferred over injectable routes like intramuscular (IM), and subcutaneous (SC) since influenza infects through mucosal tissues. Moreover, intranasal, or oral vaccine administration, can induce mucosal and cellular immune responses at the site of virus entry, thus enhancing vaccine efficacy. With regard to the experimental groups, control groups can include soluble antigen, NP vehicle, and soluble antigen with NP vehicle-with all three groups with and without adjuvant as appropriate. A PBS or sham group is also often desired to establish a background for all assays. Furthermore, a positive control group is also helpful and can be established with a commonly studied adjuvant, such as MF59® (squalene-based oil-in-water nanoemulsion) or Toll-like receptor 4 (TLR4) agonist monophosphoryl lipid A (MPL).

To analyze the humoral response of the vaccine, one may evaluate the production of specific antibodies against the influenza in response to the vaccination via antigen-specific antibody titers using ELISA assays and sera from vaccinated animals. With regard to mouse models, BALB/c mice are known to be skewed towards a Th2 response and generally often report higher total sera $\mathrm{IgG}$ compared to $\mathrm{C} 57 \mathrm{BL} / 6$ mice. To understand Th2 skewing of antibodies in a BALB/c mouse, IgG2a titers can also be evaluated (16). In addition to generalized anti-antigen titers, neutralization and hemagglutination inhibition (HAI) can be used to evaluate the efficacy of the generated titers in inhibiting viral infection. HAI can be particularly helpful in evaluating universal influenza vaccines because it can use multiple influenza strains or subtypes to illustrate activity across strains. Similarly, antibody binding to M2e or stalk sequences from various strains or subtypes can be used to identify universality of protection (16).

Besides humoral response, the cellular response of the vaccine can be evaluated. An antigen recall assay can help to determine cellular immune responses. Typically, 7-10 days after the last vaccination, draining lymph nodes and spleens can be removed from the animal and restimulated with antigen (e.g., M2e) to determine the ability of these cells to respond to the antigen they have been vaccinated against. The observation and secretion of interferon-gamma (IFN- $\gamma$ ) is the primary cytokine that is involved in antiviral responses and is a crucial player in promoting cell-mediated immunity (31), although other cytokines can also be evaluated. Cytokines can be evaluated with an ELISA, Luminex, ELISpot, RT-PCR, or flow cytometry. For more precise evaluation of which cell types are producing cytokines, cells can be presorted using magnetic beads or a flow cytometry panel can be used. Flow allows for other evaluation of important cell types such as memory cells. 


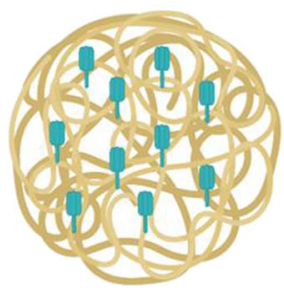

$=\mathrm{HA}$
Liposomes

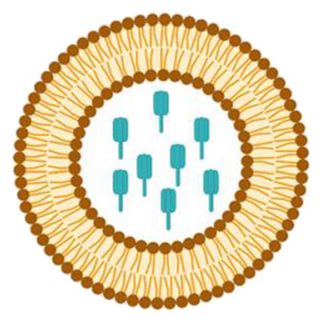

Gold NPs

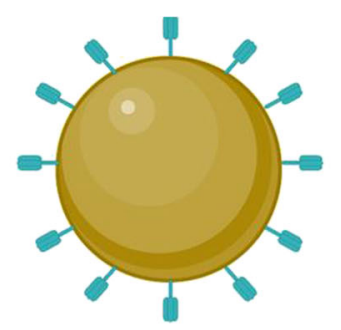

Protein Based NPs

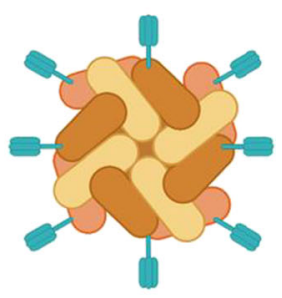

Figure 2. Summary of different nanoparticle formulations used for influenza vaccinations with HA as the model antigen. Polymeric nanoparticles are shown with antigen encapsulated throughout the polymer matrix; however, antigen can also be conjugated to the outside of polymeric nanoparticles. Liposomes are also shown with encapsulated antigen in the aqueous phase, but antigens can be coated to the outside of liposomes as well. Protein-based nanoparticles (ferritin) and gold nanoparticles are shown using surface conjugation

\section{POLYMERS}

Several polymers have been used to encapsulate vaccine elements in NPs, and the primary benefit of formulation is controlled release of encapsulated antigen and/or adjuvant. Most polymeric formulations are matrix devices wherein the encapsulates are distributed throughout the polymer, including at the polymer surface. The most common method for fabrication of polymeric NPs is via single or double emulsion with solvent evaporation. The polymers used for vaccine NPs include chitosan, PLGA, and acetalated dextran (Ace-DEX).

\section{Chitosan Nanoparticles}

Chitosan is a natural, and cationic mucoadhesive biopolymer that consists of glucosamine residues $(8,32)$. This biopolymer is derived by the partial deacetylation of chitin, which is found in the shells of crustaceans (33). Because of chitosan's unique mucoadhesive properties, it has been used as a needle-free vaccine to mucosal sites including IN, oral, and ocular $(8,32)$. Chitosan nanoparticles are commonly fabricated via an ionic gelation method. Positively charged chitosan is ionically crosslinked with a negatively charged salt (e.g., sodium tripolyphosphate, sodium citrate, sodium sulfate). Often this is performed by dripping the chitosan mixture into the salt mixture, although microfluidics and simple mixing can also be used. This method is similar for the formation of NPs out of alginate and other charged polymers.

Using ionic gelation, Dhakal et al. (8) encapsulated killed SwIAV antigen (KAg) in chitosan. The authors reported a particle size of $571.7 \mathrm{~nm}$. The $\mathrm{EE}$ of $\mathrm{KAg}$ in chitosan nanoparticles was $67 \%$ with a surface charge of $+1.69 \mathrm{mV}$. NPs were given IN using a pig model with a prime, a boost 21 days later, and a H1N1 challenge 35 days post prime vaccination. IN administration is thought to enhance mucosal immune responses which is essential in protection against influenza. Six days after challenge, the pigs were euthanized and serum and bronchoalveolar lavage (BAL) fluid were collected for analysis. The authors observed that HAI titers as well as secretory IgA antibody levels from the nasal swabs after prime-boost vaccination were higher in chitosan KAg NP-vaccinated pigs compared to those that received the soluble antigen. However, the $\mathrm{IgG}$ antibody response in the chitosan-formulated vaccine and KAg-alone vaccine was comparable. Also, Dhakal et al. reported reduced nasal virus shedding, viral lung titers, and inflammatory changes in the lungs after challenge in animals vaccinated with their chitosan and KAg NP formulation.

Hajam et al. (14) used chitosan NPs to deliver both mRNA and protein elements to generate a combined seasonal and universal influenza vaccine. The cationic nature of chitosan was used to help complex the anionic mRNA and the HA protein and M2e peptides were adsorbed on the NP surface (Figure 3). The NPs were between 100 and $800 \mathrm{~nm}$ in size. Chickens were vaccinated, at a schedule of 0 prime and 21-day boost, with a challenge at day 28 with either H7N9 or H9N2 virus. When both mRNA and protein antigens were used, the greatest lung lavage $\operatorname{IgA}$ and sera $\operatorname{IgG}$ against HA and M2e was observed. Moreover, neutralizing titer was significantly greater for mRNA and protein NP-vaccinated groups. $\mathrm{T}$ cell proliferation was measured, after antigen restimulation, and the mRNA and protein NP-vaccinated group had the highest proliferation in response to M2e and HA. Universality was displayed when chickens were challenged with H7N9 or H9N2 and illustrated increased survival over controls. Overall, the combined antigen formats facilitated by the chitosan NP delivery illustrated broad protection as a universal influenza vaccine.

\section{PLGA Particles}

Due to its biocompatibility and biodegradability, PLGA is one of the most widely used and explored polymers in vaccine and therapeutics delivery and is currently approved by the FDA and European Medicines Agency for controlled release of drugs $(15,34,35)$. Although PLGA is used extensively as vaccine carrier pre-clinically, it has not been highly studied in the influenza vaccine landscape. PLGA particles are commonly formulated via single and double emulsion solvent evaporation methods. In brief, a double emulsion solvent evaporation technique involves dissolving the polymer in an organic phase, with the antigen or water soluble adjuvant in an aqueous phase (36). An organic soluble adjuvant could be dissolved in the solvent phase with the polymer. Typically, an emulsifying agent or stabilizer is used in an emulsion, most commonly polyvinyl alcohol (PVA). One phase is suspended in the other, in the presence of the stabilizer and mixed at high speeds through sonication 

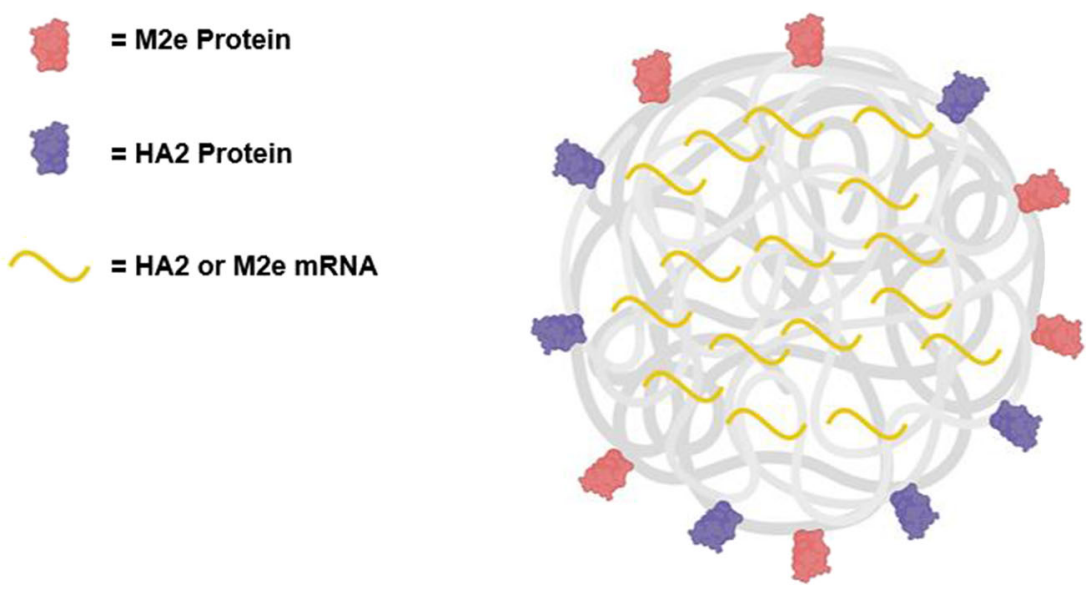

Figure 3. Protein-coated chitosan nanoparticles from Hajam et al. (14). Particles were prepared via ionic gelation. To entrap the mRNA coding for either HA2 or M2e proteins in the particles, particles were prepared at low $\mathrm{pH}$ in order to increase the positive charge of the chitosan allowing for ionic interactions with negatively charged mRNA. Furthermore, the chitosan nanoparticles were cross-linked using sodium triphosphate after the addition of the mRNA. M2e and HA2 proteins were added to the particles after crosslinking and bound to the surface via electrostatic interactions

or homogenization. For a double emulsion, the phases are mixed twice, both in the presence of a stabilizer. After the second mixing, the emulsion is stirred on a stir plate for several hours, the solvent evaporates, and particles are washed and collected via centrifugation for lyophilization and storage. For a single emulsion, the steps are the same except the encapsulates would need to be solubilized in the organic phase with the polymer and they would only be mixed once and in the presence of the stabilizer. This method can be used for many hydrophobic polymers, and denaturation of protein antigens can result from both solvent exposure and high-speed mixing (29).

Dhakal et al. (9) developed PLGA nanoparticles as a vaccine carrier with KAg. PLGA nanoparticles were prepared via the double emulsion and solvent evaporation technique wherein KAg was sonicated with PLGA and PVA in the presence of protein stabilizers sucrose and magnesium hydroxide. This primary emulsion was then sonicated again in the presence of PVA to produce the secondary emulsion which was then stirred overnight to allow for the evaporation of organic solvents. KAg-loaded PLGA NPs had a mean diameter of $313 \mathrm{~nm}$ and an encapsulation efficiency of $57 \%$. Furthermore, the reported $\zeta$-potential of the KAg particles was $-18 \pm 0.56 \mathrm{mV}$. In vitro analysis of the particles revealed $22 \%$ burst release and $27 \%$ release of the encapsulated cargo after $24 \mathrm{~h}$. The antigen showed a slow sustained release with a total cumulative release of approximately $50 \%$ after 1 month. To test the efficacy of their vaccine platform in vivo, Dhakal et al. vaccinated pigs with either soluble or PLGAencapsulated KAg. Pigs were vaccinated intranasally with these different groups and then boosted 3 weeks after the initial dose. The group vaccinated with the KAg NPs showed a significantly greater number of IFN- $\gamma$-producing cells after virus restimulation when compared to the soluble $\mathrm{KAg}$ or the mock vaccination. Furthermore, the KAg NP group demonstrated greater protection against SwIV H1N1 challenge with less fever, lower viral titers, and reduced lung lesions. Interestingly, the HAI and viral neutralization titers of the
NP group were comparable to that of the soluble antigen. The authors concluded that this is because the NPs provide protection via a cell-based immune response as evidenced by their restimulation experiments.

With regard to a universal influenza vaccine, Hiremath et al. (15) conducted a similar study fabricating PLGA NPs to encapsulate inactivated H1N1 influenza peptide antigens, M2e, and Mycobacterium vaccae whole cell lysis as an adjuvant. They used a double emulsion method to fabricate the particles. The size of the nanoparticles ranged from 227 to $316 \mathrm{~nm}$ and they report a surface charge of $-21.93 \mathrm{mV}$. The EEs of the formulations ranged from 50 to $54 \%$ with a cumulative release of $64 \%$ over a period of 4 weeks. To evaluate immune responses, Hiremath et al. vaccinated pigs IN twice at 2-week intervals and challenged 2 weeks after the boost. Interestingly, the PLGA NP formulation with and without adjuvant did not induce high antibody response. Thus, the authors concluded that $M$. vaccae is not a potent adjuvant in their vaccine. This could be a function of the NPs not distributing well in the nasal mucosa, and thereby not trafficking to the nasal associated lymph node tissues (NALT). Evaluation of the formulation as an injectable vaccine may better indicate the robustness of this platform for universal influenza vaccine development.

\section{Ace-DEX Microparticles}

Ace-DEX is an acid-sensitive biopolymer synthesized from dextran where the pendant hydroxyl groups are converted into acetal groups (10) (Figure 4). Once phagocytosed, the acid sensitivity of the polymer results in rapid intracellular release of cargo in the phagolysosome's acidic environment $(28,37,38)$. Additionally, Ace-DEX particles have been shown to be stable at elevated temperatures (37). Ace-DEX has been used in many preclinical applications, such as delivering adjuvants to enhance vaccine efficacy, therapies for Salmonella enterica infection, and scaffold-based interstitial delivery of chemotherapeutics for glioblastoma 

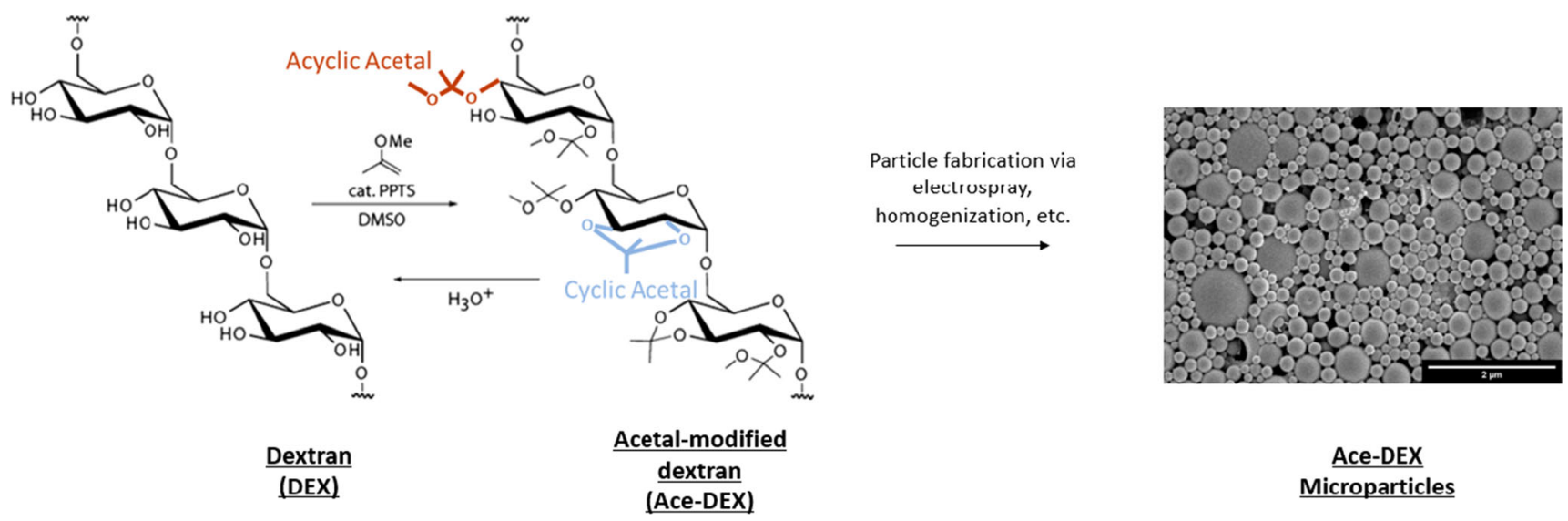

Figure 4. Synthesis of Ace-DEX. Hydroxyl groups of dextran are converted to acetal groups. This changes the hydrophilic dextran into the hydrophobic and acid-sensitive acetal-modified dextran. Ace-DEX can then be fabricated into microparticles for antigen or adjuvant delivery by processes such as homogenization or electrospraying

$(10,25,39,40)$. Particulate Ace-DEX has similar cell toxicity and viability to other biomaterials, such as PLGA and polylactic acid (PLA) (40). Ace-DEX can be fabricated into polymeric particles for vaccines using various processing methods such as sonication, homogenization, and electrospray. Electrospray is a continuous method that provides easy scalability, and better encapsulation efficiencies $(10,29,41)$. Rather than having a continuous aqueous phase-like emulsion processes, electrospray has an air continuous phase that is less likely to permit diffusion of encapsulate. Furthermore, it is performed at room-temperature and not elevated temperature like spray drying.

Junkins et al. (10) used electrosprayed Ace-DEX particles to encapsulate cGAMP as an adjuvant in a seasonal influenza vaccine. For comparative purposes, the authors encapsulated cGAMP using emulsified Ace-DEX particles, emulsified PLGA, and electrosprayed PLGA particles as well as a common liposome formulation. The diameter for the electrospray formulations were $1540 \mathrm{~nm}$ and $2890 \mathrm{~nm}$ for Ace-DEX and PLGA microparticles, respectively. The liposome formulation had the highest loading capacity, but only $30 \%$ EE, whereas electrospray Ace-DEX particles had an EE of $89.7 \%$. The electrosprayed Ace-DEX cGAMP particles illustrated the highest level of type-1 IFN production in primary dendritic cells, compared to the other formulations even after sterilization of the particles via gamma irradiation. After characterization, the particles were injected IM in C57BL/6 mice. HA (PR8) antigen was delivered as a soluble protein and mixed cGAMP particles before immunization on days 0 and 21. After vaccination, mice were challenged IN with PR8 on day 42 after prime. Antibody responses were assessed with ELISA and cellular responses were determined with ELISA and ELISpot. Electrosprayed Ace-DEX particles exhibited potent type-1 IFN cytokine response with antigen recall and a Th1 skewed humoral responses with the inclusion of cGAMP particles. Furthermore, Junkins et al. showed that their vaccine formulation protected over $80 \%$ of immunized mice against lethal influenza challenge.

Chen et al. (16) used Ace-DEX particles incorporated with M2e and cGAMP as the antigen and adjuvant to develop a universal influenza vaccine platform. Blank, M2e-, or cGAMP-loaded microparticles were fabricated with different polymer degradation profiles via a double-emulsion solvent evaporation and characterized by DLS, SEM, and an endotoxin quantification kit. The diameter ranged between 622 and $747 \mathrm{~nm}$ and EEs were over $50 \%$ for the formulations. Vaccination was given IM on day 0 and 21, with a PR8 challenge on day 42. Mice vaccinated with M2e and cGAMP particles elicited a potent humoral and cellular response and protected against the lethal challenge. Formulations with the slowest degrading particle resulted in the greatest antibody production, cellular response, and protection against challenge. Furthermore, Chen et al. found that serum antibodies from the particle formulations demonstrated cross reactivity against M2e sequences of various influenza subtypes, which can suggest broad protection, thus providing potential to develop a universal influenza vaccine.

\section{LIPOSOMES}

Liposomes are one of the most common NP formulations for therapeutics delivery; however, their use in vaccines is limited. Internationally, Inflexal $\mathrm{V}$ is an approved liposomal based influenza vaccination, where inactivated influenza virus is encapsulated in a liposome. This formulation is often commonly called a virosome. In comparison of the efficacy of Inflexal $\mathrm{V}$ to other influenza vaccines, the virosome formulation can be administered to a wider range of ages than some of the influenza vaccines currently clinically approved $(42,43)$. For the generation of liposomes, often thinfilm hydration is used at the laboratory scale. For this method, a lipid cake is formed and rehydrated with water-soluble agents included in the solution. This solution is then mixed through stirring or higher energy processes like sonication. Multilaminar liposomes are then formed to be sized and formed into unilamellar liposomes through extrusion. This method can also be facilitated through microfluidics and electrospray $(44,45)$. At the industrial scale, ethanol injection is usually performed wherein ethanol with suspended lipids is introduced into a stirred tank with the encapsulate in an aqueous phase.

For development of a seasonal influenza vaccine, BarnierQuer et al. (11) evaluated the absorption or encapsulation of HA with liposomes and delivery of the TLR agonist CpG. The liposome was generated by thin-film hydration and comprised of 1,2-diacyl-sn-glycero-3-ethylphosphocholine, dimethyl dioctadecyl- 
ammonium bromide, and $3 \beta-\left[\mathrm{N}^{-}\left(\mathrm{N}^{\prime}, \mathrm{N}^{\prime}\right.\right.$-dimethylaminoethane)carbamoyl] cholesterol. The liposomes were $155-160 \mathrm{~nm}$ in size and -46 to $-51 \mathrm{mV}$ in charge. Interestingly, the adsorbed and encapsulated HA had similar EEs at $60 \%$ and $63 \%$, respectively. C57BL/6 mice were vaccinated at days 1 and 22 through SC injection. Adsorbed HA had increased immunogenicity compared to encapsulated HA, regarding generation of titer and HAI. Inclusion of $\mathrm{CpG}$ in the liposome resulted in more efficient generation of antigen-specific responses than a liposome without CpG or an alum-based adjuvant. Overall, the platform illustrates promise for generation of a season influenza vaccine; however, a challenge was not performed to fully elucidate the differences observed.

With regard to universal influenza vaccine, Dhakal et al. (17) composed a lecithin, cholesterol, and alpha tocopherol (vitamin E) liposome to encapsulate M2e with other B and T cell peptides. These liposomes were co-delivered with the uncommon adjuvant monosodium urate (MSU). Liposomes were made through thin-film hydration and were $127-141 \mathrm{~nm}$ in diameter with a charge ranging from -25 to $-31 \mathrm{mV}$. The NPs had an EE of $52-92 \%$ depending on the hydrophobicity of the peptide sequence. Liposomes were co-delivered with MSU crystals via IN route to a pig on days 0 and 14, with a H1N1 viral challenge at day 35. Peptides delivered via a liposome with MSU crystals had lower viral shedding post challenge, but not compared to the peptide alone in solution with and without adjuvant or liposome vehicle given with the peptide. The highest frequency of T-helper and memory cells was from peptide liposomes with and without adjuvant and were significantly higher than vehicle control. Overall, the authors concluded that the MSU adjuvant did not significantly increase vaccine efficacy, but that liposomal encapsulation of peptide did enhance immune responses.

\section{METALS}

Metal-based nanoparticles can have antigen and/or adjuvant added to the carrier via chemistry that covalently attaches the antigen or adjuvant to the surface. Additionally, simple adsorption can also be used, although this is a dynamic process where proteins in particular can adsorb to and diffuse off the surface overtime. However, one benefit of surface presentation of antigens is that they allow for direct B cell interaction, which may boost antibody production. Previous studies done by others have evaluated encapsulation of protein antigen over adsorbed or surface bound antigen (11, 46-52). These studies either used liposomes $(11,48-52)$ or polymeric microparticles $(46,47)$. They concluded that surface presentation of antigen, as with the common adjuvant alum, leads to enhanced antibody titers (46). Surface presentation of antigen is one advantage of metal-based particle systems, and to evaluate the role of surface bound antigen in the generation of universal influenza vaccines, gold NPs have been used.

\section{Gold nanoparticles}

Gold NPs have been utilized in a variety of applications due to their small dimensional size, biocompatibility, and good stability. Furthermore, the material has facile synthesis routes to attach a variety of molecules to the surface (53).
Most commonly gold NPs are commercially purchased; however, they can be synthesized through reduction of chloroauric acid. Whereas polymers and liposomes can encapsulate antigen and adjuvant inside their matrix, these elements must be functionalized on the gold NP surface, usually through thiol chemistry.

Wang et al. (12) formulated an IN vaccine containing gold NPs with conjugated recombinant trimetric influenza A/Aichi/2/68 (H3N2) HA on the surface coupled with Tolllike receptor 5 (TLR5) agonist flagellin (FliC) as an adjuvant. The gold nanoparticles were prepared by reacting chloroauric acid with sodium citrate. Tween 20 was added for stability and thiol-nitrilotriacetic acid or azide-polyethylene glycol-thiol was added for the exchange of citrate with thiol. HA and FliC were then added separately to enforce the conjugation via a metal chelating reaction or click chemistry. After conjugation, TEM imaging was used to observe particle morphology and DLS confirmed the diameters of 18, 47, and $106 \mathrm{~nm}$, for gold NPs alone, NPs with FliC, or NPs with HA, respectively. Mice were immunized IN twice at 4-week intervals and challenged 4 weeks after booster with $\mathrm{H} 3 \mathrm{~N} 2$ virus. Animals vaccinated with FliC and HA gold NPs elicited a stronger systemic and mucosal humoral response compared to NPs alone and soluble antigen and adjuvant. Vaccination with this formulation induced a higher IgG2a/IgG1 ratio. Only the FliC- and HA NP-vaccinated group survived challenge and illustrated significantly greater HAI against the $\mathrm{H} 3 \mathrm{~N} 2$ virus.

Gold NPs have also been utilized for a universal influenza vaccine. Tao et al. $(19,20)$ developed an IN M2e vaccine with $\mathrm{CpG}$ as a soluble adjuvant attached to gold nanoparticles. M2e was covalently attached to the $12 \mathrm{~nm}$ gold NPs; however, soluble and covalently attached M2e was used in the vaccination. Mice vaccinated IN on day 0 and 21 with M2e conjugated NPs and CpG illustrated a dose response in serum $\mathrm{IgG}, \mathrm{IgG} 1$, and IgG2a. Similarly, a dose response was observed when mice were challenged at day 42 with PR8 wherein $100 \%$ of the mice survived the challenge. In evaluating the longevity of the vaccination, the authors report titers and survival after challenge at least 8 months after the initial vaccination. In a very similar study, the investigators illustrate that stronger immune responses were observed when $\mathrm{CpG}$ was included with the vaccination. In a subsequent paper, they used the same formulation and illustrated survival after challenge from A/California/04/2009 (H1N1) pandemic strain, A/Victoria/3/75 (H3N2), and A/Vietnam/1203/2004 (H5N1) (54).

\section{Protein-Based Nanoparticles}

Protein-based NPs have been used for influenza vaccination. These protein carriers can assemble into structures nanoscale in size including particles. An example of a proteinbased nanoparticle used for universal influenza applications is one made from ferritin.

\section{Ferritin Nanoparticles}

Ferritin is a protein which binds and stores iron and is present in most living organisms. The ferritin that can be isolated from Helicobacter pylori has been shown to self- 
assemble into an octahedron consisting of 24 subunits, with a hollow interior. Proteins can be covalently linked to the outside of ferritin particles via specific amino acid residues (i.e., aspartic acid). These residues are presented $28 \AA$ apart in groups of three with eight groups located on the outside of the NP. This spacing is identical to the spacing of trimeric HA (13).

Kanekiyo et al. (13) fused the ectodomain of A/New Caledonia/20/1999 to ferritin via the aspartic acid cluster residues, forming eight trimeric clusters on the NP (Figure 5). The recombinant proteins were expressed in $293 \mathrm{~F}$ cells. The ferritin particles alone were reported to be $14 \mathrm{~nm}$ by DLS and the addition of HA increased the diameter to $37 \mathrm{~nm}$. Mice were vaccinated on days 0 and 21 with various groups including an inactivated influenza virus vaccine (Fluzone) control. The commercially available adjuvant Ribi (Sigma) was used, which comprises of an emulsion similar to Complete Freud's Adjuvant, except instead of heat killed Mycobacterium tuberculosis TLR4 agonist MPL from Salmonella enterica Serovar Minnesota is used. MF59 was also used in some experiments. The mice vaccinated with ferritin-HA NPs illustrated significantly greater HAI and neutralizing titer when adjuvanted with Ribi or MF59, compared to Fluzone controls. Similar results were illustrated in ferrets, where viral titers of nasal washes were also shown to be significantly reduced. Additionally, antibodies isolated from serum were shown to be neutralizing against multiple H1N1 stains.

For use as a universal influenza vaccine, Corbett et al. (21) used ferritin NPs which conjugated H3 and H7 HA stalk trimers. The stalk ligated NPs were shown to be approximately $20 \mathrm{~nm}$ in diameter based on electron microscopy imaging. The structure of the stalk regions was characterized through binding to antibodies, and they confirmed that the epitopes were conserved when added to the ferritin NPs. Mice vaccinated with $\mathrm{H} 3$ or $\mathrm{H} 7$ stalk NPs at 0,4 , and 8 weeks in combination with the Ribi adjuvant illustrated high levels of serum antibody titers against their respective subtypes but only H3 NPs illustrated some cross-protective and neutralizing antibodies against H7. Additionally, the NP-vaccinated mice had $100 \%$ survival when challenged with homosubtypic strains at 4 to 8 weeks after the final boost. This platform is currently in clinical trials for evaluation of unadjuvanted $\mathrm{H} 1$ stabilized stalk ferritin NPs as an influenza vaccine (NCT03814720).

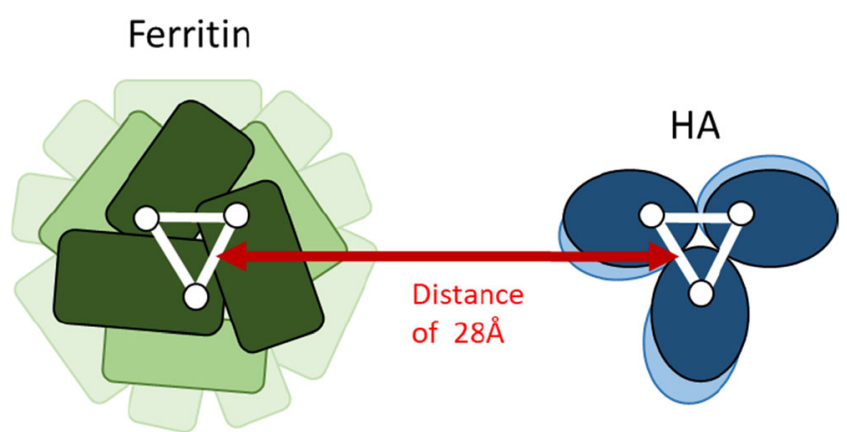

Figure 5. HA was conjugated to the aspartic acid residues found on ferritin (white dots). HA forms a trimer when conjugated to ferritin. This is because the aspartic acid residues are present in clusters of three and are $28 \AA$ apart, which is almost identical to the distance between HA monomers in the native trimer (13)

\section{CONCLUSIONS AND FUTURE WORK}

A wide breadth of nanoparticle platforms has been applied to seasonal and universal influenza vaccines. Overall, the platforms boost vaccine efficacy in the animal models they have been applied, especially in comparison to other more conventional formulations. As these platforms progress forward, it will be important to evaluate them for true universality by evaluating them against multiple heterosubtypic strains. As the field progresses, likely many of the emerging platforms that have come about to vaccinate against SARS-CoV-2 will also be applied for influenza and universal influenza antigens. These include the mRNA-based lipid nanoparticles from Pfizer and Moderna that have been FDA approved for emergency use for vaccination against COVID-19 infection (55). These platforms will likely aid in the delivery and immunogenicity of universal influenza antigens and may also help with storage outside the cold chain for better application of vaccines in resource limited settings (56-58).

A majority of the universal influenza antigens evaluated are co-formulated with adjuvants that are based on squalenebased emulsions (e.g., MF59, AS03, Ribi). Although MF59 is FDA approved in the Fluad vaccine, and squalene-based emulsions have proven to be effective inducers of potent Th2biased responses and humoral immunity, they have notable drawbacks. Alone without another adjuvant or inactivated virus, they often fail to induce significant Th1-responses that drive protective immunity against the influenza virus (59). MF59 has been shown to increase anti-HA neutralizing antibody responses (60), but studies have demonstrated that this is not necessarily correlative with protection against influenza (61). This suggests that cellular responses, which are not effectively activated by squalene emulsions (60), are also involved in protection. Also, squalene emulsions have been linked to incidences of narcolepsy $(62,63)$ and death (64). Many of the discussed formulations also include a Th1 skewing adjuvant (e.g., MPL) to help overcome many of the limitations of these emulsion adjuvants. In addition to these injected adjuvants that increase systemic immunity, mucosal adjuvants could better protect against influenza. Adjuvants which invoke strong mucosal responses, such as delta-inulin (Advax) (65) or mast cell agonists (66), may be an efficacious avenue to increase the antigenicity of universal antigens while allowing for needle-free administration, such as via the nasal route.

In addition to adjuvants, further exploration of antigens will be needed to provide effective immunity. There are concerns with $\mathrm{M} 2 \mathrm{e}$ and HA stalk as universal influenza antigens. Even though M2e reduces clinical symptoms and prevent deaths, it is unable to prevent disease (19). In addition, in a phase I clinical trial (Acambis), it was shown that M2e-specific antibody titers dropped rapidly over time, precluding future clinical development and indicating that it could not provide long-term protection (67). There are drawbacks with the stalk antigen, including that anti-stalk antibodies can enhance influenza pathogenesis (68), and they can have much lower affinity compared to other antibodies binding to HA (69). Additionally, protection generated by stalk is not necessarily through neutralizing antibodies, but through other mechanisms such as antibody-dependent cell 
mediated cytotoxicity (70). Without a high level of neutralizing antibodies, stalk vaccines protect only small animals from mortality, but does not significantly alter viral loads or lower lung inflammation $(68,71)$. Many of these challenges observed with the stalk antigen have been addressed by forming chimeric HA, where the stalk is from one subtype and the head group is from a separate subtype, even those phylogenetically different from each other (72, 73). These chimeric HAs have illustrated strong immunogenicity in clinical trials when delivered in solution with emulsion adjuvant AS03 (73).

Beyond stalk-based universal influenza vaccines, broadly active antigens may also provide protection that is superior to current season influenza vaccines. One methodology applied for antigen design is termed Computationally Optimized Broadly Reactive Antigen (COBRA). COBRA uses multiple rounds of layered consensus building to generate influenza vaccine HA antigens (74). Once identified, COBRA HA antigens elicit potent, broadly reactive HA-specific antibody responses that protect against both seasonal and novel pandemic influenza strains, including those that have undergone genetic drift (74). These vaccines induce immunity targeting both the globular head and stem regions of HA. COBRA has been used to address the diversity of $\mathrm{H} 5 \mathrm{~N} 1$ highly pathogenic avian influenza (75-79), seasonal H3N2 strains (80), and seasonal and pandemic H1N1 subtypes (74). COBRA antigens have been applied in virus-like particles, and other platforms (78).

In recent years, there have been some NP-based influenza vaccines that have moved onto clinical trials. For example, Acambis Inc. (now Sanofi Pasteur) carried out a phase I clinical trial with their NP universal influenza vaccine ACAM-FLU-A. The vaccine contains the M2e peptide fused to the capsid protein of hepatitis $\mathrm{B}$ virus $(\mathrm{HBc})$ that forms virus-like particles (VLPs) (81). The vaccine produced M2e seroconversion in $90 \%$ of the participants; however, this protection was not long lasting and dropped over a ten month period (82). More recently in 2021, the National Institute of Allergy and Infectious Disease (NIAID) Vaccine Research Center (VRC) launched a phase I clinical trial for FluMos-v1. FluMos-v1 contains HA antigens from four different serotypes displayed on a self-assembling protein nanoparticle. The advantage of this platform over others is that the protein nanoparticle presents the HA antigens in a geometrically similar way to natural influenza infection which should strengthen the immune response (83). Apart from these trials, there are many other nanoparticle-based influenza vaccines in preclinical development that have the potential to move onto clinical trials in the coming years.

Overall, the application of NPs for the formulation of universal influenza vaccines has shown to improve the efficacy of vaccinations when compared to non-formulated controls. NP-formulated vaccines have many potential advantages over non-formulated vaccines. For example, many NP formulations are able to protect cargo from degradation and clearance, target immune cells, and provide a sustained release of their cargo. Furthermore, improved universal antigens and adjuvants could enhance the efficacy of these formulations. While more research needs to be done to further investigate the universality of these vaccines as well as long-term protection, these formulations have the potential to reduce both the economic burden and mortality associated with seasonal influenza outbreaks.

\section{ACKNOWLEDGEMENTS}

Figures were created with BioRender.com

\section{AUTHOR CONTRIBUTION}

D. A. H., E. A. A., I. Y., and K. M. A. contributed to the writing of the manuscript. D. A. H. developed the figures for the manuscript. E. M. B. and K. M. A. contributed to significant editing of the manuscript.

\section{FUNDING}

This work was funded by NIH NIAID R01AI147497 and Collaborative Influenza Vaccine Innovation Centers (CIVICs) Center for Influenza Vaccine Research in HighRisk Populations contract number 75N93019C00052.

\section{DECLARATIONS}

Conflict of Interest The authors declare no competing interests.

\section{REFERENCES}

1. McElhaney JE, Andrew MK, McNeil SA. Estimating influenza vaccine effectiveness: evolution of methods to better understand effects of confounding in older adults. Vaccine. 2017;35(46):6269-74.

2. Grohskopf LA, Sokolow LZ, Broder KR, Walter EB, Bresee JS, Fry AM, Jernigan DB. Prevention and control of seasonal influenza with vaccines: recommendations of the advisory committee on immunization practices - United States, 2017-18 Influenza Season. MMWR Recomm Rep. 2017;66(2):1-20.

3. Types of Influenza Viruses. Centers for disease control and prevention, centers for disease control and prevention. https:// www.cdc.gov/flu/about/viruses/types.htm. Accessed 2 Nov 2021.

4. A revision of the system of nomenclature for influenza viruses: a WHO memorandum. Bull World Health Organ. 1980;58(4):585-91.

5. Nachbagauer R, Krammer F. Universal influenza virus vaccines and therapeutic antibodies. Clin Microbiol Infect. 2017;23(4):222-8.

6. Pati R, Shevtsov M, Sonawane A. Nanoparticle vaccines against infectious diseases. Front Immunol. 2018;9:2224.

7. Zhao L, Seth A, Wibowo N, Zhao CX, Mitter N, Yu C, Middelberg APJ. Nanoparticle vaccines. Vaccine. 2014;32(3):327-37.

8. Dhakal S, Renu S, Ghimire S, Shaan Lakshmanappa Y, Hogshead BT, Feliciano-Ruiz N, Lu F, HogenEsch H, Krakowka S, Lee CW, Renukaradhya GJ. Mucosal immunity and protective efficacy of intranasal inactivated influenza vaccine is improved by chitosan nanoparticle delivery in pigs. Front Immunol. 2018;9:934.

9. Dhakal S, Hiremath J, Bondra K, Lakshmanappa YS, Shyu DL, Ouyang K, Kang KI, Binjawadagi B, Goodman J, Tabynov K, Krakowka S, Narasimhan B, Lee CW, Renukaradhya GJ. Biodegradable nanoparticle delivery of inactivated swine influenza virus vaccine provides heterologous cell-mediated immune response in pigs. J Control Release. 2017;247:194-205. 
10. Junkins RD, Gallovic MD, Johnson BM, Collier MA, WatkinsSchulz R, Cheng N, David CN, McGee CE, Sempowski GD, Shterev I, McKinnon K, Bachelder EM, Ainslie KM, Ting JPY. A robust microparticle platform for a STING-targeted adjuvant that enhances both humoral and cellular immunity during vaccination. J Control Release. 2018;270:1-13.

11. Barnier-Quer C, Elsharkawy A, Romeijn S, Kros A, Jiskoot W. Adjuvant effect of cationic liposomes for subunit influenza vaccine: influence of antigen loading method, cholesterol and immune modulators. Pharmaceutics. 2013;5(3):392-410.

12. Wang C, Zhu W, Luo Y, Wang BZ. Gold nanoparticles conjugating recombinant influenza hemagglutinin trimers and flagellin enhanced mucosal cellular immunity. Nanomedicine. 2018;14(4):1349-60.

13. Kanekiyo M, Wei C-J, Yassine HM, McTamney PM, Boyington JC, Whittle JRR, et al. Self-assembling influenza nanoparticle vaccines elicit broadly neutralizing H1N1 antibodies. Nature. 2013;499(7456):102-6.

14. Hajam IA, Senevirathne A, Hewawaduge C, Kim J, Lee JH. Intranasally administered protein coated chitosan nanoparticles encapsulating influenza H9N2 HA2 and M2e mRNA molecules elicit protective immunity against avian influenza viruses in chickens. Vet Res. 2020;51:1-17.

15. Hiremath J, Kang KI, Xia M, Elaish M, Binjawadagi B, Ouyang $\mathrm{K}$, Dhakal S, Arcos J, Torrelles JB, Jiang X, Lee CW, Renukaradhya GJ. Entrapment of H1N1 influenza virus derived conserved peptides in plga nanoparticles enhances $\mathrm{T}$ cell response and vaccine efficacy in pigs. PLoS One. 2016;11(4):e0151922.

16. Chen N, Gallovic MD, Tiet P, Ting JP, Ainslie KM, Bachelder EM. Investigation of tunable acetalated dextran microparticle platform to optimize M2e-based influenza vaccine efficacy. J Control Release. 2018;289:114-24.

17. Dhakal S, Cheng X, Salcido J, Renu S, Bondra K, Lakshmanappa YS, Misch C, Ghimire S, Feliciano-Ruiz N, Hogshead B, Krakowka S, Carson K, McDonough J, Lee CW, Renukaradhya GJ. Liposomal nanoparticle-based conserved peptide influenza vaccine and monosodium urate crystal adjuvant elicit protective immune response in pigs. Int $\mathrm{J}$ Nanomedicine. 2018;13:6699-715.

18. Adler-Moore J, Munoz M, Kim H, Romero J, Tumpey T, Zeng H, Petro C, Ernst W, Kosina S, Jimenez G, Fujii G. Characterization of the murine Th2 response to immunization with liposomal M2e influenza vaccine. Vaccine. 2011;29(27):4460-8.

19. Tao W, Ziemer KS, Gill HS. Gold nanoparticle-M2e conjugate coformulated with $\mathrm{CpG}$ induces protective immunity against influenza A virus. Nanomedicine (Lond). 2014;9(2):237-51.

20. Tao W, Gill HS. M2e-immobilized gold nanoparticles as influenza A vaccine: role of soluble M2e and longevity of protection. Vaccine. 2015;33(20):2307-15.

21. Corbett KS, Moin SM, Yassine HM, Cagigi A, Kanekiyo M, Boyoglu-Barnum S, et al. Design of nanoparticulate group 2 influenza virus hemagglutinin stem antigens that activate unmutated ancestor B cell receptors of broadly neutralizing antibody lineages. mBio. 2019;10(1):e02810-8.

22. Genito C, Batty C, Bachelder E, Ainslie K. Considerations for size, surface charge, polymer degradation, co-delivery, and manufacturability in the development of polymeric particle vaccines for infectious diseases. Advanced NanoBiomed Research. 2021;1(3):2000041.

23. Chen L, Simpson JD, Fuchs AV, Rolfe BE, Thurecht KJ. Effects of surface charge of hyperbranched polymers on cytotoxicity, dynamic cellular uptake and localization, hemotoxicity, and pharmacokinetics in mice. Mol Pharm. 2017;14(12):4485-97.

24. Champion JA, Walker A, Mitragotri S. Role of particle size in phagocytosis of polymeric microspheres. Pharm Res. 2008;25(8):1815-21.

25. Johnson MM, Collier MA, Hoang KV, Pino EN, GrahamGurysh EG, Gallovic MD, Zahid MSH, Chen N, Schlesinger L, Gunn JS, Bachelder EM, Ainslie KM. In vivo and cellular trafficking of acetalated dextran microparticles for delivery of a host-directed therapy for Salmonella enterica Serovar Typhi Infection. Mol Pharm. 2018;15(11):5336-48.

26. Chen N, Johnson MM, Collier MA, Gallovic MD, Bachelder EM, Ainslie KM. Tunable degradation of acetalated dextran microparticles enables controlled vaccine adjuvant and antigen delivery to modulate adaptive immune responses. J Control Release. 2018;273:147-59.

27. Moore KM, Batty CJ, Stiepel RT, Genito CJ, Bachelder EM, Ainslie KM. Injectable, ribbon-like microconfetti biopolymer platform for vaccine applications. ACS Appl Mater Interfaces. 2020;12(35):38950-61.

28. Broaders KE, Cohen JA, Beaudette TT, Bachelder EM, Frechet JM. Acetalated dextran is a chemically and biologically tunable material for particulate immunotherapy. Proc Natl Acad Sci U S A. 2009;106(14):5497-502.

29. Gallovic MD, Schully KL, Bell MG, Elberson MA, Palmer JR, Darko CA, et al. Acetalated dextran microparticulate vaccine formulated via coaxial electrospray preserves toxin neutralization and enhances murine survival following inhalational Bacillus Anthracis Exposure. Adv Healthc Mater. 2016;5:261727.

30. Kim Y-J, Lee Y-T, Kim M-C, Lee Y-N, Kim K-H, Ko E-J, et al. Cross-protective efficacy of influenza virus M2e containing virus-like particles is superior to hemagglutinin vaccines and variable depending on the genetic backgrounds of mice. Front Immunol. 2017;8:1730.

31. La Gruta NL, Turner SJ. T cell mediated immunity to influenza: mechanisms of viral control. Trends Immunol. 2014;35(8):396402.

32. Dehghan A, Shahsavandi S, Jabalameli L. Improvement efficacy of influenza nanovaccine in combination with hemokinin-1 molecular adjuvant. Avicenna J Med Biotechnol. 2018;10(4):208-13.

33. Illum L, Jabbal-Gill I, Hinchcliffe M, Fisher AN, Davis SS. Chitosan as a novel nasal delivery system for vaccines. Adv Drug Deliv Rev. 2001;51(1-3):81-96.

34. Seth A, Ritchie FK, Wibowo N, Lua LH, Middelberg AP. Noncarrier nanoparticles adjuvant modular protein vaccine in a particle-dependent manner. PLoS One. 2015;10(3):e0117203.

35. Alkie TN, Yitbarek A, Taha-Abdelaziz K, Astill J, Sharif S. Characterization of immunogenicity of avian influenza antigens encapsulated in PLGA nanoparticles following mucosal and subcutaneous delivery in chickens. PLoS One. 2018;13(11):e0206324.

36. McCall RL, Sirianni RW. PLGA nanoparticles formed by single- or double-emulsion with vitamin E-TPGS. J Vis Exp. 2013;82:51015.

37. Bachelder EM, Beaudette TT, Broaders KE, Dashe J, Frechet JM. Acetal-derivatized dextran: an acid-responsive biodegradable material for therapeutic applications. J Am Chem Soc. 2008;130(32):10494-5.

38. Kauffman KJ, Do C, Sharma S, Gallovic MD, Bachelder EM, Ainslie KM. Synthesis and characterization of acetalated dextran polymer and microparticles with ethanol as a degradation product. ACS Appl Mater Interfaces. 2012;4(8):4149-55.

39. Bachelder EM, Pino EN, Ainslie KM. Acetalated dextran: a tunable and acid-labile biopolymer with facile synthesis and a range of applications. Chem Rev. 2017;117(3):1915-26.

40. Graham-Gurysh E, Moore KM, Satterlee AB, Sheets KT, Lin FC, Bachelder EM, Miller CR, Hingtgen SD, Ainslie KM. Sustained delivery of doxorubicin via acetalated dextran scaffold prevents glioblastoma recurrence after surgical resection. Mol Pharm. 2018;15(3):1309-18.

41. Steipel RT, Gallovic MD, Batty CJ, Bachelder EM, Ainslie KM. Electrospray for generation of drug delivery and vaccine particles applied in vitro and in vivo. Mater Sci Eng C Mater Biol Appl. 2019;105:110070.

42. Herzog C, Hartmann K, Kunzi V, Kursteiner O, Mischler R, Lazar $\mathrm{H}$, et al. Eleven years of Inflexal V-a virosomal adjuvanted influenza vaccine. Vaccine. 2009;27(33):4381-7.

43. Kreuter J, Liehl E. Long-term studies of microencapsulated and adsorbed influenza vaccine nanoparticles. J Pharm Sci. 1981;70(4):367-71.

44. Duong AD, Sharma S, Peine KJ, Gupta G, Satoskar AR, Bachelder EM, Wyslouzil BE, Ainslie KM. Electrospray encapsulation of toll-like receptor agonist resiquimod in polymer microparticles for the treatment of visceral leishmaniasis. Mol Pharm. 2013;10(3):1045-55. 
45. Duong AD, Collier MA, Bachelder EM, Wyslouzil BE, Ainslie KM. One step encapsulation of small molecule drugs in liposomes via electrospray-remote loading. Mol Pharm. 2016;13(1):92-9.

46. Zhang W, Wang L, Liu Y, Chen X, Liu Q, Jia J, Yang T, Qiu S, $\mathrm{Ma}$ G. Immune responses to vaccines involving a combined antigen-nanoparticle mixture and nanoparticle-encapsulated antigen formulation. Biomaterials. 2014;35(23):6086-97.

47. Shen H, Ackerman AL, Cody V, Giodini A, Hinson ER, Cresswell P, Edelson RL, Saltzman WM, Hanlon DJ. Enhanced and prolonged cross-presentation following endosomal escape of exogenous antigens encapsulated in biodegradable nanoparticles. Immunology. 2006;117(1):78-88.

48. Tan L, Weissig V, Gregoriadis G. Comparison of the immune response against polio peptides covalently-surface-linked to and internally-entrapped in liposomes. Asian Pac J Allergy Immunol. 1991;9(1):25-30.

49. Therien HM, Lair D, Shahum E. Liposomal vaccine: influence of antigen association on the kinetics of the humoral response. Vaccine. 1990;8(6):558-62.

50. Watson DS, Endsley AN, Huang L. Design considerations for liposomal vaccines: influence of formulation parameters on antibody and cell-mediated immune responses to liposome associated antigens. Vaccine. 2012;30(13):2256-72.

51. Shahum E, Therien HM. Correlation between in vitro and in vivo behaviour of liposomal antigens. Vaccine. 1994;12(12):1125-31.

52. Guan HH, Budzynski W, Koganty RR, Krantz MJ, Reddish MA, Rogers JA, Longenecker BM, Samuel J. Liposomal formulations of synthetic MUC1 peptides: effects of encapsulation versus surface display of peptides on immune responses. Bioconjug Chem. 1998;9(4):451-8.

53. Cabuzu D, Cirja A, Puiu R, Grumezescu AM. Biomedical applications of gold nanoparticles. Curr Top Med Chem. 2015;15(16):1605-13.

54. Tao W, Hurst BL, Shakya AK, Uddin MJ, Ingrole RS, Hernandez-Sanabria M, et al. Consensus M2e peptide conjugated to gold nanoparticles confers protection against H1N1, $\mathrm{H} 3 \mathrm{~N} 2$ and $\mathrm{H} 5 \mathrm{~N} 1$ influenza A viruses. Antiviral Res. 2017;141:62-72.

55. Batty CJ, Heise MT, Bachelder EM, Ainslie KM. Vaccine formulations in clinical development for the prevention of severe acute respiratory syndrome coronavirus 2 infection. Adv Drug Deliv Rev. 2021:169:168-89.

56. Alberer M, Gnad-Vogt U, Hong HS, Mehr KT, Backert L, Finak G, Gottardo R, Bica MA, Garofano A, Koch SD, FotinMleczek M, Hoerr I, Clemens R, von Sonnenburg F. Safety and immunogenicity of a mRNA rabies vaccine in healthy adults: an open-label, non-randomised, prospective, first-in-human phase 1 clinical trial. Lancet. 2017;390(10101):1511-20.

57. Stitz L, Vogel A, Schnee M, Voss D, Rauch S, Mutzke T, Ketterer T, Kramps T, Petsch B. A thermostable messenger RNA based vaccine against rabies. PLoS Negl Trop Dis. 2017;11(12):e0006108.

58. Curevac's COVID-19 Vaccine Candidate, CVnCoV, Suitable for Standard Fridge Temperature Logistics. CureVac. https:// www.curevac.com/en/2020/11/12/curevacs-covid-19-vaccine-candidate-cvncov-suitable-for-standard-fridge-temperature-logistics/. Accessed 12 Nov 2020.

59. Bungener L, Geeraedts F, Ter Veer W, Medema J, Wilschut J, Huckriede A. Alum boosts TH2-type antibody responses to whole-inactivated virus influenza vaccine in mice but does not confer superior protection. Vaccine. 2008;26(19):2350-9.

60. Calabro S, Tritto E, Pezzotti A, Taccone M, Muzzi A, Bertholet S, de Gregorio E, O'Hagan DT, Baudner B, Seubert A. The adjuvant effect of MF59 is due to the oil-in-water emulsion formulation, none of the individual components induce a comparable adjuvant effect. Vaccine. 2013;31(33):3363-9.

61. Impagliazzo A, Milder F, Kuipers H, Wagner MV, Zhu X, Hoffman RM, et al. A stable trimeric influenza hemagglutinin stem as a broadly protective immunogen. Science (New York, NY). 2015;349(6254):1301-6.
62. Nohynek H, Jokinen J, Partinen M, Vaarala O, Kirjavainen T, Sundman J, Himanen SL, Hublin C, Julkunen I, Olsén P, Saarenpää-Heikkilä O, Kilpi T. AS03 adjuvanted AH1N1 vaccine associated with an abrupt increase in the incidence of childhood narcolepsy in Finland. PLoS One. 2012;7(3):e33536.

63. Kim WJ, Lee SD, Lee E, Namkoong K, Choe KW, Song JY, Cheong HJ, Jeong HW, Heo JY. Incidence of narcolepsy before and after MF59-adjuvanted influenza A(H1N1)pdm09 vaccination in South Korean soldiers. Vaccine. 2015;33(38):4868-72.

64. Italy Suspends Fluad Flu Vaccine from Novartis after Deaths. BBC News, BBC. https://www.bbc.com/news/world-europe30240620. Accessed 28 Nov 2014.

65. Gordon DL, Sajkov D, Honda-Okubo Y, Wilks SH, Aban M, Barr IG, Petrovsky N. Human Phase 1 trial of low-dose inactivated seasonal influenza vaccine formulated with Advax ${ }^{\mathrm{TM}}$ delta inulin adjuvant. Vaccine. 2016;34(33):3780-6.

66. Choi HW, Chan C, Shterev ID, Lynch HE, Robinette TJ, Johnson-Weaver BT, Shi J, Sempowski GD, Kim SY, Dickson JK, Gooden DM, Abraham SN, Staats HF. Identification of novel mast cell activators using cell-based high-throughput screening. SLAS Discov. 2019;24(6):628-40.

67. Deng L, Cho KJ, Fiers W, Saelens X. M2e-based universal influenza A vaccines. Vaccines (Basel). 2015;3(1):105-36.

68. Khurana S, Loving CL, Manischewitz J, King LR, Gauger PC, Henningson $J$, et al. Vaccine-induced anti-HA2 antibodies promote virus fusion and enhance influenza virus respiratory disease. Sci Transl Med. 2013;5(200):200ra114.

69. Hoa LNM, Mai LQ, Bryant JE, Thai PQ, Hang NLK, Yen NTT, Duong TN, Thoang DD, Horby P, Werheim HFL, Fox A. Association between hemagglutinin stem-reactive antibodies and influenza A/H1N1 virus infection during the 2009 pandemic. J Virol. 2016;90(14):6549-56.

70. Yassine HM, Boyington JC, McTamney PM, Wei CJ, Kanekiyo M, Kong WP, et al. Hemagglutinin-stem nanoparticles generate heterosubtypic influenza protection. Nat Med. 2015;21(9):106570.

71. Valkenburg SA, Mallajosyula VV, Li OT, Chin AW, Carnell G, Temperton N, et al. Stalking influenza by vaccination with prefusion headless HA mini-stem. Sci Rep. 2016;6:22666.

72. Hai R, Krammer F, Tan GS, Pica N, Eggink D, Maamary J, Margine I, Albrecht RA, Palese P. Influenza viruses expressing chimeric hemagglutinins: globular head and stalk domains derived from different subtypes. J Virol. 2012;86(10):5774-81.

73. Nachbagauer R, Feser J, Naficy A, Bernstein DI, Guptill J, Walter EB, Berlanda-Scorza F, Stadlbauer D, Wilson PC, Aydillo T, Behzadi MA, Bhavsar D, Bliss C, Capuano C, Carreño JM, Chromikova V, Claeys C, Coughlan L, Freyn AW, et al. A chimeric hemagglutinin-based universal influenza virus vaccine approach induces broad and long-lasting immunity in a randomized, placebo-controlled phase I trial. Nature Medicine. 2021;27(1):106-14.

74. Carter DM, Darby CA, Lefoley BC, Crevar CJ, Alefantis T, Oomen R, Anderson SF, Strugnell T, Cortés-Garcia G, Vogel TU, Parrington M, Kleanthous H, Ross TM. Design and characterization of a computationally optimized broadly reactive hemagglutinin vaccine for H1N1 influenza viruses. J Virol. 2016;90(9):4720-34.

75. Crevar CJ, Ross TM. Elicitation of protective immune responses using a bivalent H5N1 VLP vaccine. Virol J. 2008;5:131.

76. Giles BM, Bissel SJ, Craigo JK, Dealmeida DR, Wiley CA, Tumpey TM, et al. Elicitation of anti-1918 influenza virus immunity early in life prevents morbidity and lower levels of lung infection by 2009 pandemic H1N1 influenza virus in aged mice. J Virol. 2012;86(3):1500-13.

77. Giles BM, Bissel SJ, Dealmeida DR, Wiley CA, Ross TM. Antibody breadth and protective efficacy are increased by vaccination with computationally optimized hemagglutinin but not with polyvalent hemagglutinin-based $\mathrm{H} 5 \mathrm{~N} 1$ virus-like particle vaccines. Clin Vaccine Immunol. 2012;19(2):128-39.

78. Giles BM, Ross TM. A computationally optimized broadly reactive antigen (COBRA) based H5N1 VLP vaccine elicits 
broadly reactive antibodies in mice and ferrets. Vaccine. 2011;29(16):3043-54.

79. Monto AS. Seasonal influenza and vaccination coverage. Vaccine. 2010;28(Suppl 4):D33-44.

80. Wong TM, Allen JD, Bebin-Blackwell AG, Carter DM, Alefantis T, DiNapoli J, et al. Computationally optimized broadly reactive hemagglutinin elicits hemagglutination inhibition antibodies against a panel of H3N2 influenza virus cocirculating variants. J Virol. 2017;91(24):e01581-17.

81. Fiers W, De Filette M, El Bakkouri K, Schepens B, Roose K, Schotsaert M, et al. M2e-based universal influenza A vaccine. Vaccine. 2009;27(45):6280-3.
82. Deng L, Wang BZ. A perspective on nanoparticle universal influenza vaccines. ACS Infect Dis. 2018;4(12):1656-65.

83. Boyoglu-Barnum S, Ellis D, Gillespie RA, Hutchinson GB, Park YJ, Moin SM, Acton OJ, Ravichandran R, Murphy M, Pettie D, Matheson N, Carter L, Creanga A, Watson MJ, Kephart S, Ataca S, Vaile JR, Ueda G, Crank MC, et al. Quadrivalent influenza nanoparticle vaccines induce broad protection. Nature. 2021;592(7855):623-8.

Publisher's Note Springer Nature remains neutral with regard to jurisdictional claims in published maps and institutional affiliations. 